Mots. Les langages du politique

\title{
La construction de l'ethos présidentiel dans le débat télévisé français
}

Francesca Cabasino

\section{OpenEdition}

Journals

Édition électronique

URL : https://journals.openedition.org/mots/18743

DOI : $10.4000 /$ mots. 18743

ISSN : 1960-6001

Éditeur

ENS Éditions

Édition imprimée

Date de publication : 30 mars 2009

Pagination : 11-23

ISBN : 978-2-84788-151-6

ISSN : 0243-6450

Référence électronique

Francesca Cabasino, "La construction de l'ethos présidentiel dans le débat télévisé français », Mots. Les langages du politique [En ligne], 89 | 2009, mis en ligne le 30 mars 2011, consulté le 22 avril 2022. URL : http://journals.openedition.org/mots/18743; DOI : https://doi.org/10.4000/mots. 18743 


\section{La construction de l'ethos présidentiel dans le débat télévisé français}

Ayant choisi comme objet d'observation la période de cristallisation de la campagne française pour la présidentielle (mars 2007), nous allons essayer, d'abord, d'identifier les dominantes argumentatives des propos tenus dans l'espace télévisuel par trois candidats qui incarnent, chacun à sa manière, un processus de rupture et souhaitent obtenir l'adhésion d'un public hétérogène convié à s'exprimer politiquement dans les urnes. Le discours argumenté adopte alors comme norme l'efficacité, puisqu'il est destiné à «faire bien voter » plutôt qu'à «faire croire». Nous reprenons ici les mots de Christian Plantin qui précise que «les catégories de la persuasion, de la vérité, de la croyance sont subordonnées aux problématiques du faire» (1996, p. 19). Si argumenter c'est influencer, agir sur le destinataire par la parole signifie, comme le souligne Patrick Charaudeau (2004, p. 167), tenter de «modifier un état mental» préexistant et voir l'intention suivie d'effet. Dans le cas d'une élection aussi décisive, le consensus portera sur le besoin de se reconnaitre dans les valeurs communes mobilisées dans les discours prononcés.

Il s'agira ensuite de pénétrer dans ce que les conversationnalistes appellent des négociations d’identité, des situations susceptibles d'évoluer, de se réajuster, selon la perspective goffmanienne, au cours de la relation interlocutive et de fournir en temps réel des éléments indispensables pour la réception.

Car, malgré l'intention manifeste de dépasser les affrontements rituels et de concrétiser le modèle de l'action communicative théorisé dans les ouvrages du philosophe Habermas, c'est-à-dire l'intercompréhension, les émissions sont conçues en fonction des attentes des téléspectateurs et des instances médiatiques. Celles-ci sont vouées à faire ressortir non seulement la crédibilité d'un discours lié à une dimension promissive et donc soumis à une double vérification portant sur les conditions de sincérité de l'énonciateur et sur ses capacités à obtenir des résultats positifs, mais aussi tout ce que le comportement du politique présente d'implicite, de paradoxal, d'insolite et d'émotionnel.

Or, si l'on examine les séquences d'ouverture lors desquelles a lieu cette lutte verbale particulière, on constate deux phénomènes étroitement liés.

Faculté des Sciences politiques, Université de Rome Sapienza, francesca.cabasino@uniroma1.it 
D'une part, le commentateur de presse donne en connaisseur, puisqu'il fréquente depuis longtemps les responsables politiques, sa perception de l'interviewé, vise à fixer une identité aux contours flous, à la redéfinir selon ses propres convictions, à créer un véritable masque symbolique et propose aux téléspectateurs des critères selon lesquels il est possible de juger les actes et les comportements de l'acteur politique. De l'autre, ce dernier, qui a acquis, avec l'exercice du métier, une aptitude à discerner le danger de certaines prises de position, peut habilement rejeter les mises en doute ou les présupposés négatifs que l'interlocuteurveut fonder sur la notion de vérité.

L'échange sera donc caractérisé par des rapports d’influence déterminés par les jeux stratégiques des interactants : Bourdieu (1996, p. 38) parle à ce propos de «complicité entre professionnels».

Dans la situation de production trilogale (journaliste-invité-public) qui caractérise toute interaction médiatique, les spectateurs - présents ou absents sur le plateau, silencieux ou actifs - constituent les véritables «destinataires du discours échangé » (Kebrat-Orecchioni, Plantin, 1995, p. 22) et soulignent l'aspect théâtral du dispositif télévisuel. On examinera donc encore une fois (Cabasino, 1992, p. 29-49) le couple question/réponse, quand l'exercice dynamique de l'interrogation, expression d'un pouvoir légitime, devient envahissant et tente de modifier des opinions et des valeurs reçues en influençant les interprétations de l'auditoire.

\section{Un discours d'action}

Nous partirons donc de la stratégie de prise de contact déployée par Arlette Chabot, directrice de l'information de France 2, le 8 mars 2007, pour faire découvrir la personnalité d'un invité qui est reçu pour la deuxième fois en quatre mois sur le plateau de $A$ vous de juger :

A. Chabot. - Alors je veux vous demander / c'est dur une campagne présidentielle?1

N. Sarkozy - (pensif, les yeux vers le bas). - oui (signe affirmatif de la tête, débit lent)

il faut s’y préparer / il faut y avoir pensé / il faut être prêt à assumer la pression (souriant) parce que la pression de la campagne / ce sont les prémisses / des pressions de la fonction

A. Chabot. - oui / pourtant vous étiez effectivement bien préparé / c'est encore (mouvement des mains) plus difficile que vous n'aviez imaginé?

N. Sarkozy. - non

1. La ponctuation particulière de ces citations, de même que l'absence de majuscules en début de phrase, font partie des conventions de transcription. En caractères gras : accentuation des segments verbaux; deux points (:) : allongement syllabique; entre parenthèses et en italique : commentaires du transcripteur (rires, modulations de la voix, débit rapide ou ralenti, etc.) ; mot coupé d'un trait d'union (dis-parition) : scansion; slash (/) : pause brève; double slash (//) : pause longue; crochet ouvert ([) : chevauchement. 
A. Chabot. - non ? et alors c'est pour ça que de temps en temps on dit / tiens il est un peu nerveux / il est tendu (modulations de la voix) / ça dépend de quoi / des sondages / de l'atmosphère / du moment (intonation semi-assertive)

N. Sarkozy. - bon / je sais pas d'abord / je peux pas empêcher les commentaires bien sûr / mais vous imaginez quand même pas qu'on vient à la télévision parler à des millions de Français / (débit lent, intonation neutre) qui sont en train de faire un choix / pour une élection / absolument décisive / sans y avoir réfléchi? / je veux d'ailleurs dire à ces Français que moi j'ai / bien l'intention de tenir ma promesse / dire la vérité / la France (yeux rivés sur A. Chabot) a besoin de changements profonds / la France peut faire aussi bien que les autres / la France peut avoir le plein-emploi / c'est possible la promotion sociale / et je veux porter le changement / (mouvements affirmatifs de la tête) je m'y suis préparé / j'aurai une ma-jorité pour cela et si les Français (mouvements de la tête vers l'avant) me font confiance / tout ce que j'aurai dit avant l'élection / je le respecterai scrupuleusement après l'élection.

L'acte initiatif contraignant - «c'est dur une campagne présidentielle?» - vise manifestement à faire réagir et sollicite une évaluation immédiate sur la position inconfortable d'un candidat à la présidence. Un énoncé apparemment bref reçoit dans ce contexte une accentuation prononcée en raison de la structure utilisée, sans inversion, donc plutôt familière, avec une intonation montante fortement marquée. Cependant, le processus de réception s'engage lentement; Nicolas Sarkozy réfléchit avant de répondre, comme l'indiquent les éléments paraverbaux relevés, qui confirment la mise en scène d'une tactique défensive. Il s'efforce en effet de structurer son identité et réussit à satisfaire la curiosité de la journaliste en appuyant son argumentation sur la modalité déontique et sur un mouvement rythmique en trois temps qui fait penser à l'élocution gaullienne (Cabasino, 1983). On peut remarquer l'énergie du troisième enchainement argumentatif - «il faut être prêt à assumer la pression » correspondant à la conscience aigüe d'un candidat responsable de son dire. S'appuyant sur une relation causale, il entend communiquer au public l'importance d'un argument pragmatique nécessaire pour formuler une première conclusion relative aux risques que comporte l'exercice du pouvoir. Aux présomptions d'Arlette Chabot, il semble opposer un comportement coopératif, signe qu'il accepte une négociation sur les contenus et légitime en quelque sorte la question posée. Ce qui serait prouvé également par la mimique faciale souriante du candidat de l'UMP, attentif dès le début de l'interview à présenter une image crédible, sereine et non conflictuelle.

On connait l'impact des stratégies d'insistance et des procédés d'insinuation sur l'interaction et le rôle ambigu du journaliste, lien de contact avec le spectateur et détenteur d'un droit de critique qu'il exerce, dans la dynamique de médiatisation, malgré une politesse de surface (Cabasino, 1992 et 1998). Or, la relance impromptue d'Arlette Chabot au sujet des impressions suscitées dans la classe médiatique par un présidentiable en proie à l'anxiété, 
associée à des modulations de la voix et des allongements vocaliques, montre une tendance à proposer des points de vue personnels identifiables à l'opinion commune. Malgré ses compétences en matière politologique, la responsable de l'information de France 2 n'échappe pas à un comportement habituel chez les professionnels de la presse et qu'il est convenu d'appeler, après les recherches psychologiques de Ghiglione, "stratégie de harcèlement» (1990, p. 134). Cela provoque une réaction de Nicolas Sarkozy sous forme de question rhétorique, où l'ironie subtile sous-jacente se cache derrière un ton neutre, sans écarts, et un débit ralenti et contrôlé, manifestations indirectes des convictions du locuteur. En déplaçant un objet de discours imposé, l'interviewé remet en cause une question jugée insignifiante et engage la discussion sur l'enjeu de sa candidature. II introduit ensuite des modalités révélatrices de son implication dans le discours, des verbes de parole clairement assumés comme «je veux dire à ces Français que moi j'ai bien l'intention de tenir ma promesse, dire la vérité», ou des formules explicitant une volonté d'action - «je veux porter le changement» - et aptes à obtenir le consensus parce qu'il laisse entendre que le mouvement, l'innovation sont prévus au sein des institutions. Comme on peut l'observer, les intentions performative et promissive alternent avec une nécessité perlocutoire, car le candidat entend renforcer le lien avec des électeurs potentiels.

Le choix du pronom personnel de première personne n'exclut pas des dénominations moins directes où le locuteur s'identifie à d'autres sujets présents dans les énoncés, notamment à «la France », susceptible de réagir à une situation de crise. Et cette sorte de dédoublement du responsable politique, propre au style gaullien, ne semble pas un procédé emphatique (Cabasino, 1983), mais un moyen pour établir encore une fois le contact avec l'auditoire.

La présence et l'accentuation des modalités aléthiques dans l'énonciation des objectifs à atteindre, parmi lesquels l'élimination de la précarité, signifient que pour lui le plein emploi n'est pas une illusion et que la France et ses dirigeants ont la capacité de réaliser les conditions pour défendre un modèle social valable aussi au niveau européen. Interpréter ce besoin de changement équivaut par ailleurs à expliciter et à renforcer l'un des slogans qui scandent sa campagne, «Ensemble tout devient possible», parce que le rapport entre le politique et les citoyens ne peut être qu'un rapport de «confiance».

Le dernier acte de promesse de la séquence, accentué par l'insertion de l'adverbe scrupuleusement, se veut la confirmation d'un ethos² obsédé par l'engagement, une sorte de «rationalité stratégique» qui surdétermine au niveau sémiotique la communication et fonctionne comme une «compétence épistémique» fondée sur ses propres intentions et ses désirs (Parret, 1990, p. 59). En définissant sa stratégie opérationnelle, Nicolas Sarkozy vise

2. Sur la notion d'« ethos oratoire», voir Amossy (2000, p. 60-85). 
à actualiser une relation intersubjective stable avec les téléspectateurs. En outre, cette image de soi projetée vers l'engagement qui le fait reconnaitre comme acteur social coexiste avec un ethos de crédibilité, présupposé indispensable pour que les Français comprennent qu'il a le pouvoir d'agir efficacement et s'en remettent à lui pour les guider dans une situation difficile.

Dans la confrontation avec des invités qui clôt le format de cette émission, le 8 mars, l'ex-candidat écologiste Nicolas Hulot devient le destinataire privilégié du candidat au cours des dernières minutes du débat. Déçu par le comportement d'une classe politique insensible aux problèmes de l'environnement et par les promesses réitérées de l'interviewé, il n'hésite pas à l'interrompre :

N. Hulot. - [Un mot / Nicolas Sarkozy / moi / je voudrais simplement là vous livrer deux secondes mon cœur / moi / la planète (mouvement des mains parallèles) / la biodiversité / la réalité des / des changements climatiques / c'est ma seule culture / c'est pas une abstraction / je vois la planète agoniser / je dis simplement que le $21^{\mathrm{e}}$ siècle c'est le siècle de tous les dangers et que : on sera comptable parce que : / jusqu'à présent on savait pas / maintenant nous savons / donc à partir de maintenant vous et moi nous sommes responsables / donc c'est pour moi le (débit ralenti) grand enjeu universel de ce siècle et je pense que dans les ambitions qui sont les vôtres vous / devez / garder / cette ambition / cette mission comme la / grande priorité

N. Sarkozy. - mais vous savez / Arlette Chabot / c'est pas d'aujourd'hui que je discute avec Nicolas Hulot de ces questions-là / il le sait parfaitement bien / si je n'étais pas convaincu de cela / vous savez je ne suis pas un homme qui pratique le mensonge / la langue de bois et le double langage / j'ai beaucoup de défauts et je ne prétends pas avoir la vérité (doigt pointé) et je vous dis quelque chose / quand je suis convaincu qu'un enjeu est majeur pour notre pays / et cet enjeu du développement / durable est majeur / je me bat-trai pour que vous voyiez dans les faits (rond de la main) que cette conviction qui est la vôtre / c'est aussi la mienne.

$\mathrm{Si}$, dans ce passage, Nicolas Hulot communique ses préoccupations profondes et vise à motiver son combat pour une réalité contemporaine incontournable, il faitallusion égalementau rapportcomplexe qui le lieà son interlocuteur, semble rejeter une logique de séduction purement électorale et revenir à une des thématiques centrales du discours politique, l'attribution de responsabilité. L'accentuation de certains segments - «je vois la planète agoniser» -, ou le débit ralenti à des moments cruciaux de son énonciation - «c'est pour moi le grand / enjeu universel de ce siècle »-, tous ces facteurs langagiers et prosodiques se fondent sur une sorte de véracité dramatisante qui transmet une grande efficacité à son dire. Mais cette conscience de soi ne peut se former qu'à travers une action concertée avec l'autre. Il tente alors d'impliquer l'homme politique dans ses croyances et dans son système de pensée en lançant un appel pressant à la responsabilité : «vous et moi nous sommes responsables». Il s'agit dans ce cas d'une double responsabilité : individuelle et sociale. L'habileté persuasive 
qu'il met en œuvre consiste à associer l'ambition, qui est le ressort de tout candidat, et la mission délicate dans laquelle ce dernier est engagé. On peut se demander cependant si ce rapprochement est voulu, c'est-à-dire s'il constitue une superposition de concepts ou un simple lapsus.

La réponse incisive du futur président, qui souligne l'effective entente des points de vue, adressée indirectement à son destinataire par l'intermédiaire d'Arlette Chabot et donc fondée sur un mécanisme de distanciation, explicité par le pronom personnel de la troisième personne, fait partie d'une mise en scène fonctionnelle à la recherche du consensus, car elle porte sur une thématisation explicite destinée à retenir l'attention de l'auditoire. L'image de soi que le présidentiable entend construire se modèle en termes de valeurs et d'interdits et se fonde sur la dimension axiologique (refus du mensonge, du double langage, de discours insignifiants et vides), mais parallèlement semble représenter une critique implicite des actes d'autorité. Même s’il reconnait que la vérité est parfois inaccessible, il ne peut se passer d'évoquer ce que les philosophes anglo-saxons appellent les «conditions de sincérité » des conduites langagières. Sa promesse vouée à soutenir une action en faveur du développement durable, si elle sort des formules habituelles exprimant l'engagement et si elle reçoit une accentuation par la force des paroles associée aux composantes intonatives et gestuelles, correspond aux objectifs de transformation du monde dont il se sent investi. Habile partisan du pragmatisme ainsi qu'excellent communicateur, il met en relief l'importance de cette expérience intersubjective et, ce qui est le plus intéressant, en propose une évaluation partagée. C'est ainsi que, au cours de l'interaction, les participants perçoivent et négocient les enjeux de leur rencontre.

\section{Une nouvelle réalité à construire}

Sorte de contrepoids au débat électoral qui se déroule parallèlement, ces fragments d'une interview située dans le contexte d'une émission culturelle, Esprits libres du 9 mars 2007, en disent long sur un projet politique à peine esquissé et sur les moyens choisis par l'animateur pour impliquer l'interlocuteur et le faire réagir. Guillaume Durand, tout en privilégiant une dimension imaginaire fondée sur des présomptions et une expérience personnelle de journaliste politique, définit son champ d'action à travers la création d'un système de représentations et de références.

Voyons comment l'ouverture de l'émission est révélatrice des stratégies mises en œuvre pour construire l'image de François Bayrou, candidat de l'UDF:

G. Durand. - Projet d'avenir / donc un livre qui regroupe l'essentiel des propositions faites pour les Français et qui est publié aux éditions Plon / / de Gaulle / Mendès / Merkel c'est un programme rêvé? (mouvement circulaire de la main à la hauteur de la tête) 
F. Bayrou. - pourquoi rêvé?

G. Durand. - je ne sais pas

F. Bayrou. - ça n'a rien d'un rêve / c'est au contraire une réalité à construire / désormais / possible et plausible et / cherchant l'effet qui / Pierre Mendès-France le disait dans les mêmes mots / a tourné la page / sur quelque chose / sur vingtcinq années qui ont été à mon sens vingt-cinq années pour la France négatives / en tout cas qui ont plongé la France dans une impasse / maintenu la France dans une impasse

G. Durand. - ah voilà on va plonger dans le livre et vous avez dit vingt-cinq années de choses négatives // c'est justement l'argument (main ouverte vers l'interlocuteur) que les sarkozystes d'un côté et les royalistes de l'autrevous assignent // comment peut-il dénoncer vingt-cinq années d'incurie et au fond dire je vais / comme ce qu'on vient de voir / gouverner avec eux ben / je sais pas / dans / dans deux mois

F. Bayrou. - (ferme, mais avec calme) pas avec eux

G. Durand. - avec qui?

F. Bayrou. - avec // les sensibilités profondes du peuple français qui accepteront / comme / ça vient d'être dit / qui accepteront (il se frotte lentement les mains) de se regrouper pour redresser le pays autour d'objectifs (ronds des mains en geste de précision) je ne veux pas dire simples / presque sim-plissimes / qui sont les objectifs normaux et naturels

G. Durand. - [et comment ils vont venir?

F. Bayrou. - d'un peuple / d'un peuple qui décide qu'il va désormais regarder en face (paumes vers le haut et vers l'interlocuteur) les problèmes qui sont les siens

G. Durand. - [mais comment

F. Bayrou. - et y répondre / et au lieu / de perpétuellement mettre en scène (les deux paumes parallèles tournées vers le haut) l'affrontement joué (les index pointés vers le haut) / l'affrontement artificiel (mouvement des mains) entre deux clans qui au fond veulent conserver le monopole du pouvoir / au contraire / on va répondre à tous les problèmes démocratiques de la det-te / (scansion des mots simultanée à un mouvement rythmique des mains sur la table, de gauche à droite) de l'éducation / de l'exclusion / de l'emploi avec des mesures sur lesquelles on se sera mis d'accord

G. Durand. - [mais

F. Bayrou. - alors / comment vont-ils venir? dites-vous

G. Durand. - ah voilà / c'est ça / le grand argument qui vous est adressé (débit rapide) on se dit d'une façon / imaginons (mouvement large des bras) même qu'il soit élu / comment les hiérarques de tous les partis vont travailler avec lui / ils ont fait campagne contre lui

F. Bayrou. - [je ne demande pas les hiérarques / je ne demande pas les hiérarques

G. Durand. - [et ils continueront

F. Bayrou. - [je pense qu'il faut changer les visages et changer de génération / alors y a des gens bien / ceux-là auront leur place / pourquoi viendront-ils? parce que le peuple français / leur en aura / donné l'instruction / à partir du moment où le peuple français donne mandat / en élisant un président de la République / en mobilisant la majorité (tronc vers l'avant, paumes vers le haut) absolue des citoyens pour dire nous voulons que ça change / que voulez-vous que fassent les appareils? 
Dès la présentation, François Bayrou apparait dans le rôle de l'écrivain qui a bousculé l'actualité politique après la publication de son essai sur le projet d'une grande coalition entre les principaux partis français. Un projet évocateur de ressemblances avec l'action menée dans l'histoire récente par deux personnalités «légendaires», de Gaulle et Mendès-France. Et l'habileté de Guillaume Durand consiste à glisser dans ses propos un terme désignatif, rêvé, destiné à définir, à étiqueter, à cerner la personnalité du candidat.

Le questionnement commence donc par une négociation explicite qui est déjà interprétative - «c'est un programme rêvé?»-, où le débit rapide, l'accentuation du qualificatif et le mouvement qui l'accompagne clarifient l'intention de provoquer l'interlocuteur. La réponse du candidat par une autre question indique que l'interrogation est jugée inacceptable et exige une réfutation qui ne tardera pas à se manifester. Le débit ralenti met alors en relief une tentative de persuasion adressée au public-témoin et vise à conduire le débat sur un plan plus rationnel.

Mais, comme on l'a observé dans d'autres interactions analogues (Cabasino, 1992), l'insistance du journaliste consiste aussi à s'approprier les mots des autres - dans ce cas, des antagonistes du candidat - pour mettre le politique en contradiction avec lui-même. Cependant, face à cet agir stratégique, la réfutation est immédiate. Conscient d'avoir à convaincre un auditoire diversifié, le représentant de l'UDF tente d'obtenir le consensus en présentant comme une «réalité à construire » l'objectif de redresser la France et en montrant que, malgré les clivages politiques, les Français peuvent encore se reconnaitre dans des valeurs partagées. L'interpellation indirecte du «peuple» (ce terme est employé quatre fois dans la séquence) constitue en effet une stratégie de persuasion apte à concrétiser une image, certes simplifiée, du destinataire. Cette tentative d'implication du public est associée à des accentuations, des scansions et des gestes de précision révélateurs de l'engagement mental et corporel de l'énonciateur. Cela est confirmé par les nombreux efforts, non réussis, d'interruption de la part de Guillaume Durand, signe que dans la partie centrale de l'interview le président de l'UDF est la figure dominante. Et cette position haute se manifeste également dans les reprises, les anticipations, les énoncés brefs adressés à l'interlocuteur comme alors, comment vont-ils venir? dites-vous, qui poussent celui-ci à proposer de nouveau son point de vue. L'impératif imaginons représente en effet une sorte d'injonction négative que le journaliste emprunte aux adversaires de François Bayrou et inspire encore des doutes sur la réussite de son action politique.

La longue réponse du candidat centriste est fondée sur deux éléments essentiels : la nécessité d'un rajeunissement radical de la classe politique, explicitée par un nous voulons englobant qui vise à impliquer encore davantage les téléspectateurs, et la construction d'une image personnelle d'honnêteté contre les appareils et les querelles partisanes. À travers ces arguments clairs, 
François Bayrou s'impose à son interlocuteur privilégié et en même temps aux citoyens qui sont invités à adhérer aux croyances et valeurs proposées.

\section{«Je veux incarner le changement "}

La troisième séquence d'ouverture dont nous allons nous occuper concerne l'interview que Ségolène Royal accorde à Arlette Chabot dans le cadre de l'émission «À vous de juger» du 15 mars 2007.

A. Chabot. - J'ai d'abord envie de vous demander est-ce que / vous aviez / bien mesuré la difficulté d'une campagne présidentielle?

S. Royal. - (respiration profonde) c'est une campagne difficile bien évidemment / très difficile même / parce que c'est un poids / immense / qui pèse/ sur mes épaules / c'est en même temps / un moment exaltant parce que j'ai conscience qu'une page de l'histoire de France / est en train de s'écrire / que je représente euh / l'espoir de millions et de millions d'électeurs qui ont envie que ça change et que ça change euh / vraiment (tête vers le bas) et que je veux / (respiration) incarner ce changement pour que la France reprenne la main (mouvements de la tête vers l'avant) [...] je veux que cette élection serve à quelque chose [...] et le choix qui sera fait par les Français dans quelques semaines va définir notre avenir (mouvement affirmatif de la tête) commun [...]

A. Chabot. - alors il y a des gens qui disent / on la reconnait pas bien Ségolène Royal depuis quelque temps / parce qu'on la voyait / li-bre (modulations de la voix) / elle disait des choses qui pouvaient / paraitre extrêmement auda-cieuses pendant justement cette campagne au parti socialiste / mais depuis quelques semaines (débit rapide) on dirait que / vous êtes un peu plus cade-nassée alors est-ce que c'est le poids du parti socialiste (modulations de la voix et mouvements de la tête) / est-ce que c'est la peur du dérapage/ est-ce que vous êtes allez / je veux pas dire pas sous contrôle /je ne me permettrais pas / mais un peu coincée beaucoup plus et vous avez perdu cette liberté (intonation semiassertive)

S. Royal. - (respiration) c'est un peu vrai (mouvement affirmatif de la tête) je crois que j'ai eu beaucoup de liberté dans la / le débat / interne du parti socialiste [...] puis j'ai eu cette phase / d'écoute parce que je veux / répondre à la crise démocratique et je crois qu'aujourd'hui on ne (mouvements négatifs de la tête) peut pas faire de la politique comme avant / la France a changé / le monde a changé / la politique donc doit changer / les citoyens sont devenus exi-geants (la caméra cadre Jack Lang qui passe la main sur la bouche et touche sa cravate) / ils se sont souvent détournés des urnes et cette crise / cette attente / ces colères j'ai voulu les entendre / avant de proposer ce / ce pacte / présidentiel

A. Chabot. - [vous êtes moins libre maintenant ou pas alors?

S. Royal. - j'ai été moins libre c'est vrai / parce que je crois que je suis / appuyée par une organisation politique et par d'autres partis

A. Chabot. - c'est un poids quand même / ça vous aide quand même aussi S. Royal. - c'est une chance / c'est une chance parce que ça prépare aussi la stabilité du pouvoir pour après / dans le cadre d'une majorité parlementaire / mais en 
même temps il a fallu que / que je compose dans cette phase / mais aujourd'hui je suis dans la dernière ligne / droite et je reprends / Arlette Chabot / je reprends toute ma liberté.

On peut remarquer immédiatement que l'orientation de la première question ne s'éloigne pas du schéma thématique imposé à Nicolas Sarkozy - la difficulté d'une campagne présidentielle -, mais ici l'approche est beaucoup plus insinuante. L'allusion porte en effet sur le fait que le candidat est une femme mue par l'ambition d'arriver à un poste de grande responsabilité et polarise l'attention sur le terme d'adresse vous suivi de l'adverbe bien et d'un prédicat choisi pour sa charge d'évaluation. Une relation de dominance va donc se construire car la journaliste exige une réponse précise de son destinataire exclusif en laissant entrevoir le déséquilibre des positions symboliques et les âpretés de tout dialogue qui se veut spontané.

La respiration profonde de Ségolène Royal est la première réaction à cette intrusion dans la sphère personnelle, mais la perception d'un léger embarras initial est vite effacée par l'effort de répondre aux attentes des électeurs. La reprise des paroles de l'autre permet à la représentante du Parti socialiste de souligner dès le début les raisons de sa candidature et le double sentiment qui l'anime, la prise de conscience des difficultés et l'exaltation due au nouveau virage imprimé à la politique. Les mots sélectionnés et le rythme énonciatif mettent en relief un dessein ambitieux et clarifient sa vision des choses. "Je veux incarner ce changement » n'est pas seulement une promesse soutenue par une volonté tenace, c'est, grâce à la force sémantique du verbe employé, une tentative vigoureuse de dominer la situation et de souligner la valeur de vérité de cette rupture. Ce qui sera explicité plus loin dans l'appel aux Français pour surmonter un état de crise qui vise à susciter des réflexes sociaux, à solliciter des appréciations ou des réserves, à fournir des stimuli pour prolonger la discussion, mais surtout à renégocier la place de quelqu'un qui rêve d'offrir une nouvelle chance à ses compatriotes.

Si la journaliste, dans la suite du dialogue, semble imposer à sa partenaire un contexte défavorable par les opinions indirectement avancées à propos de l'audace initiale de Ségolène Royal et de la «liberté cadenassée» d'aujourd'hui, c'est qu'elle fait un usage stratégique du questionnement ou plutôt des assertions camouflées sous forme d'interrogation. Elle suggère donc un certain nombre d'implicites et derrière l'apparence d'une triple alternative, proposée en respectant les règles de politesse, mais en utilisant des modulations intonatives, elle laisse planer le doute sur cette absence de liberté dans la période actuelle.

Sur le plan de l'énonciation, la candidate ne se pose pas le problème de la pertinence de la question, mais entend en clarifier les présupposés en justifiant la longueur d'une phase d'écoute avant de signer son programme. Le jeu mouvant de l'argumentation et l'insistance sur le je qui précède la modalisa- 
tion de la volonté sont associés à une expressivité du visage nettement marquée, à des mouvements affirmatifs ou négatifs de la tête, à des accentuations multiples, à des scansions qui rythment ses phrases.

Parfois la journaliste de France 2 n'hésite pas à suggérer à la candidate certains termes pour définir la situation inconfortable qu'elle va affronter au sein de son parti - «c'est un poids quand même / ça vous aide quand même aussi» -, mais Ségolène Royal n'accepte pas des suggestions ambigües et juge nécessaire une opération de recadrage de la question et de mise à distance d'un discours imposé. On assiste donc à ce que Goffman (1987) a nommé l'ajustement des identités : à travers une négociation constante, la candidate du PS refuse une identité attribuée qui met en jeu sa crédibilité et revendique la fonction primaire d'un présidentiable voué à renouer «un lien direct» avec «le peuple français ». En même temps, Ségolène Royal réaffirme sa cohérence, ses valeurs dans la tentative de récupérer une position haute qu'elle sent menacée. Sa façon de s'adresser à la journaliste - «je reprends, Arlette Chabot, je reprends toute ma liberté »-, et notamment la présence du nom propre, indique à la fois un effet de clôture, sorte de commentaire aux insinuations, et le renforcement d'un discours d'autorité qui rétablit l'exercice de la liberté dont les commentateurs de presse l'ont provisoirement privée.

Au-delà des contraintes imposées et d'une ritualisation inévitable, les documents oraux examinés demeurent le lieu privilégié où s'élaborent des comportements discursifs, métadiscursifs et paralangagiers qui transforment profondément la parole politique. Si le sens du discours se construit dans l'espace interactif quand le sujet communicant exploite un matériau verbal disponible ou crée de nouvelles formulations, il ne faut pas négliger la composante situationnelle - dans ce cas le cadre médiatique - qui amplifie le résultat des paroles ou met à nu le comportement humain.

L'analyse de discours envisagée a permis de découvrir des éléments significatifs dans les longues négociations qui opposent journalistes et candidats : des effets de mise en scène des objets langagiers comme la «dureté» de la campagne, le "rêve» d'une coalition inaccessible ou encore les "valeurs» du «changement» et d'une nouvelle éthique présentées aux électeurs, aux réactions des invités, face à des jugements de cohérence parfois sollicités à propos de paroles ou d'attitudes antérieures. Souvent coincé par des questions insinuantes et contraint dans un contexte de force (voir l'allusion réitérée à la «liberté» perdue de Ségolène Royal), le responsable politique fait tous ses efforts pour refuser la thématique imposée et pour renverser le rapport dominant-dominé afin de pouvoir présenter aux téléspectateurs une image de soi responsable et crédible.

Les trois ethos ici mis en lumière ne s'éloignent pas des figures classiques faisant partie des typologies proposées par des chercheurs qui s'occupent 
d'analyse de discours (Amossy, 2000, Charaudeau, 2005): la crédibilité fondée sur des conditions de sincérité, le refus de l'idéologie et un potentiel de réussite pour Nicolas Sarkozy, la solidarité envisageant une attention à l'écoute des citoyens pour Ségolène Royal, l'honnêteté préconisée par François Bayrou, image moins stéréotypée que les deux précédentes, vouée à conquérir un nouvel espace politico-éthique impliquant la mise à l'écart des appareils, où les jeunes générations pourraient se reconnaitre. La modalisation de la volonté, accentuée par la présence d'un je en tension, rapproche les candidats de droite et de gauche, tandis que le représentant de l'UDF, en privilégiant le nous et l'appel au peuple, tente de mobiliser directement les citoyens.

Or, le candidat qui construit, au cours des émissions en question, une image proche du rôle présidentiel et coïncidant avec les représentations que se font les Français du chef de l'État à l'époque contemporaine est quelqu'un qui sait retravailler son ethos préalable et tenir compte des attentes de l'imaginaire collectif. Peut-on alors adopter, à propos du futur élu, la formule de Parret relative au «stratège-joueur» (1990, p. 62) ? Celui qui sait faire ses calculs et réussit à séduire les électeurs par ses appels à la confiance, ses idées claires sur la ligne à suivre et sa capacité à dominer les appareils, mais qui respecte en même temps le discours et les sentiments de l'autre (notamment dans la séquence avec Nicolas Hulot).

\section{Références}

Amossy Ruth, 2000, L'argumentation dans le discours, Paris, Nathan.

Bourdieu Pierre, 1996, Sur la télévision, Dijon, Liber éditions.

CABAsino Francesca, 1983, Malraux e de Gaulle. Enunciazione e argomentazione nel discorso memorialistico, Rome, Bulzoni.

- 1992, L'interview politique télévisée. Conflits, métadiscours, paralangage, Rome, EuRoma.

- 1995, "L'interaction médiatique comme construction du sens», M. Margarito, A. M. Raugei éd., Studi di linguistica, storia della lingua, filologia francesi, Alessandria, Edizioni dell'Orso, p. 63-77.

- 1998, «Due candidate alla presidenza della Repubblica francese tra discorso e antidiscorso », LiSt, $\mathrm{n}^{\circ}$ 4-5, p. 129-150.

- 2007, Poly-logiques. Méthodologie de l'interaction pluri-locuteurs dans l'espace télévisuel, Rome, EuRoma.

Charaudeau Patrick, 2004, "Comment le langage se noue à l'action dans un modèle socio-communicationnel du discours. Del'action au pouvoir», Cahiers de linguistique française, $\mathrm{n}^{\circ} 26$, p. 151-175.

- 2005, Le discours politique. Les masques du pouvoir, Paris, Vuibert.

GHIGLIONE Rodolphe et al., 1990, " “Écoute voir" ou le discours politique médiatisé», Psychologie française, $\mathrm{n}^{\circ} 35$ (2), p. 123-142. 
Goffman Erving, 1987, Façons de parler, Paris, Minuit.

Kerbrat-Orecchion Catherine, Plantin Christian, 1995, Le trilogue, Lyon, Presses universitaires lyonnaises.

PARRET Herman, 1990, "La rationalité stratégique», L'interaction communicative, H. Parret, A. Berrendonner éd., Berne, Peter Lang, p. 47-69.

Perelman Chaim, Olbrechts-TyteCA Lucie, 1958, Traité de l'argumentation. La nouvelle rhétorique, Paris, PUF.

Plantin Christian, 1996, L'argumentation, Paris, Le Seuil. 